\section{Mathematics in Nature}

M athematical structures occur throughout naturefrom honeycombs and ammonites to the geometry of crystals and snowflakes.

The Fibonacci sequence features in the patterns on sunflowers and pinecones. It is related to the logarithmic spiral, found on ammonites and snail shells, which was first studied mathematically by René Descartes and later by Jacob Bernoulli.
As liquids crystallize they assume the form of various polyhedra: fluorite crystals appear as octahedra, while lead and zinc sulfide crystals appear as cuboctahedra and truncated tetrahedra.

There are three regular tiling patterns of the plane, formed by equilateral triangles, squares, and regular hexagons. The hexagonal arrangement was discussed by the Greek mathematician Pappus and appears naturally in the form of a bee's honeycomb.

The delicate structure of snowflakes has sixfold rotational symmetry-rotation by $60^{\circ}$ leaves the pattern unchanged. Their hexagonal form was recognized by the Chinese in the second century $\mathrm{BC}$ and was later investigated by Kepler, Descartes, and others.

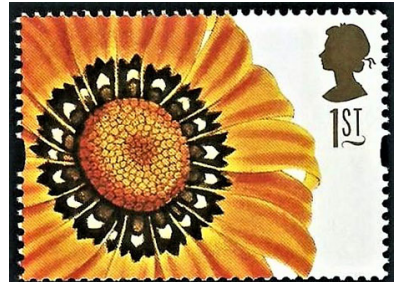

Sunflower

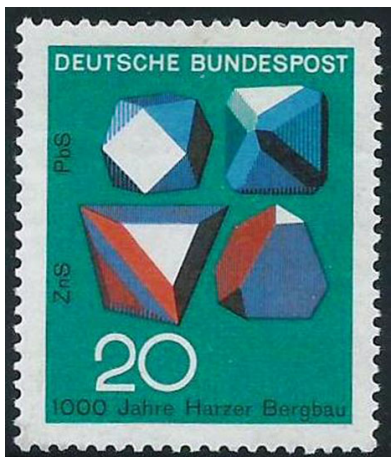

Sulfide crystals

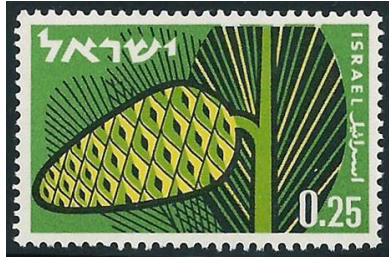

\section{Pinecone}

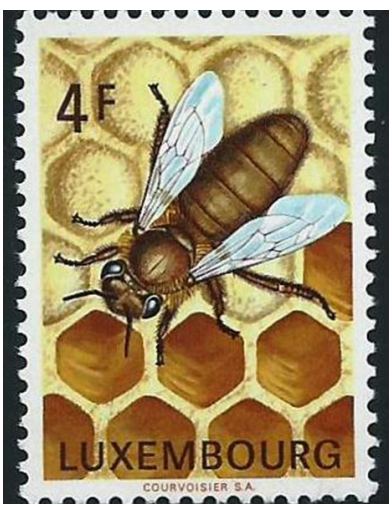

Honeycomb
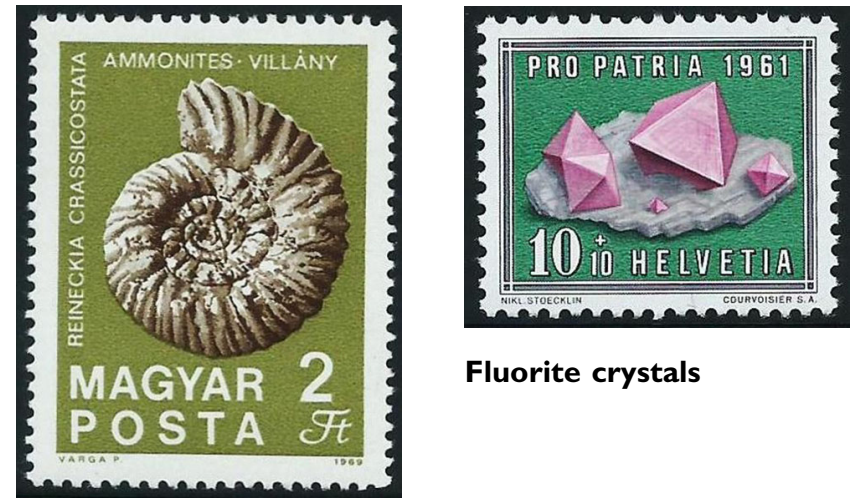

Fluorite crystals

\section{Ammonite}

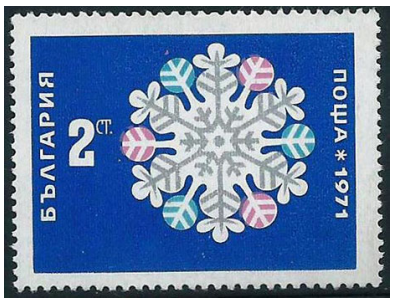

Snowflake I

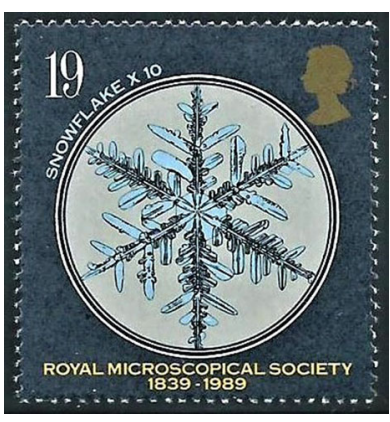

Snowflake 2

$>$ Robin Wilson

Publisher's note Springer Nature remains neutral with regard to jurisdictional claims in published maps and institutional affiliations. 